\title{
MULTI OBJECTIVE AERODYNAMIC OPTIMIZATION USING PARALLEL NASH EVOLUTIONARY/DETERMINISTIC HYBRID ALGORITHMS
}

\author{
ZHILI TANG \\ College of Aerospace Engineering, Nanjing University of Aeronautics and Astronautics \\ 29 Yudao Street, Nanjing, 210016, China \\ tangzhili@nuaa.edu.cn
}

Published 22 June 2016

\begin{abstract}
This paper solved aerodynamic drag reduction of transport wing fuselage configuration in transonic regime by using a parallel Nash evolutionary/deterministic hybrid optimization algorithm. Two sets of parameters are used, namely globally and locally. It is shown that optimizing separately local and global parameters by using Nash algorithms is far more efficient than considering these variables as a whole.
\end{abstract}

Keywords: Nash equilibrium; hybridized evolutionary/deterministic algorithms; multi objective aerodynamic optimization.

\section{Introduction}

Since Nash ${ }^{1,2}$ introduces Nash Equilibrium (NE) theory in the early 50's, it has become an efficient tool to solve Multi Objective Optimization (MOO) problems in aerodynamics ${ }^{3,4,5,6}$ and other relative fields ${ }^{7,8}$. The solution of a MOO problem can be viewed as a NE under the concept of competitive games. This paper proposed an evolutionary/deterministic hybrid algorithm for calculating the NE. A NE is reached when each player, constrained by the strategy of the others, cannot improve further its own criterion.

In many MOO problems, multiple objectives are not always in the same property. For instance, some objectives are continuous and differentiable functionals, whereas some are discontinuous and/or discrete functionals. In addition, some objectives can be optimized easily by using Evolutionary Algorithms (EAS), and others are more suitable by using Deterministic Algorithms (DAs), refer to gradient based method.

All the optimization algorithms have advantages and disadvantages. The gradientbased (deterministic) algorithms usually converge quickly in the vicinity of the solution, and are therefore very interesting in terms of rapidity. However, they have some limitations, being strongly dependent on user skills, due to the need to choose the initial

This is an Open Access article published by World Scientific Publishing Company. It is distributed under the terms of the Creative Commons Attribution 3.0 (CC-BY) License. Further distribution of this work is permitted, provided the original work is properly cited. 
trial solutions. Also, they can easily fall to local minimums. The requirement of derivative calculation makes these methods non-trivial to implement.

EAs is a population-based optimization algorithm that makes use of some mechanism to improve the initial solutions. The trial solutions to the optimization problem are individuals in a population. Evolution of the population takes place after the repeated application of the genetic operators (selection, crossover, mutation, etc.). Although they are robust methods, their convergence is very time-consuming, and they must be considered as sub-optimal algorithms, as for continuous variable optimization the global minimum of the objective function is not guaranteed. Anyway, local minima are generally avoided and the final solution is in the vicinity of the global minimum.

So, the idea in this paper is to bring together evolutionary and deterministic algorithms and combining the advantages of each approach in order to solve complex MOO problem efficiently under the concept of Nash game. The proposed methodology is tested and evaluated for aerodynamic drag reduction of transport wing fuselage configuration in transonic regime. Two sets of parameters are used, namely globally and locally. The global characteristics of the wing shape are dihedral position and twist angle of each section. The local characteristics of the wing shape are defined by imposing the section shape. The mixing of global and local parameters leads to complex interactions among multi-modality. The change in the shape of a given wavelength produces changes of the same wavelength in the solution. Thus, while the high-frequency changes in the shape produce changes in the solution that are of high frequency and remain locally to the neighborhood of the shape, the smooth (low-frequency) changes in the shape produce smooth changes to the solution and are global in nature. It is shown that optimizing separately local and global parameters by using Nash algorithms is far more efficient than considering these variables as a whole.

The remainder of this paper is organized as follows. In the section 2, the basic knowledge from the game theory is presented, and a Nash EAs/DAs hybrid algorithm for calculating the NE is proposed. Section 3 solves an aerodynamic drag reduction of a civil transport wing fuselage configuration by using the proposed algorithms. Finally a short conclusion is given in Section 4.

\section{Physical Model and Numerical Method}

Since Nash introduced the fundamental concept of equilibrium point, Nash equilibrium has become an efficient tool to solve multi objective aerodynamic optimization problems. Here, a parallel algorithm to calculate the NE by using hybrid Evolutionary/Deterministic methods is presented.

\subsection{Definition of a Nash equilibrium}

Let $N$ be the number of players. Each player $v \in\{1, \ldots, N\}$ controls the variables $x_{v} \in \mathbf{R}^{n_{v}}$. Let $x=\left\{x_{1}, \ldots, x_{N}\right\}^{T} \in X \subseteq \mathbf{R}^{n}$ be the vector formed by all these decision variables, where $n:=n_{1}+\ldots+n_{N} \geq N$. To emphasize the $v t h$ player's variables within the vector $x$, we sometimes write $x=\left(x_{v}, x_{-v}\right)^{T}$, where $x_{-v}$ subsumes all the other players' variables. 
Let $\tau_{v}:=\mathbf{R}^{n} \mapsto \mathbf{R}$ be the $v$ th player's payoff (or loss) function. Consider the following constraint MOO problem since practical aerodynamic optimization problems usually involve constraints, such as drag reduction under fixed lift, etc.

$$
\begin{aligned}
& \min _{x_{v}} \tau_{v}(x), v=1, \ldots, N \\
& \text { subject to } g(x) \leq 0, h_{v}\left(x_{v}\right) \leq 0
\end{aligned}
$$

The interest in games that allow for constraints in the combined search space is Generalized Nash Equilibrium (GNE) ${ }^{9}$.

Often, GNE Problem (GNEP) can be reduced to a classical NE Problem (NEP), once the set $X$ is given by a set of inequalities like

$$
X=\left\{x \in R^{n} \mid g(x) \leq 0, h_{v}\left(x_{v}\right) \leq 0, \forall v=1, \ldots, N\right\}
$$

for some functions $g:=\mathbf{R}^{n} \mapsto \mathbf{R}^{m}$ and $h_{v}:=\mathbf{R}^{n_{v}} \mapsto \mathbf{R}^{m_{v}}$ with some numbers $m, m_{v} \geq 0$. Here $g$ represents the global constraints of all players, whereas $h_{v}$ depends only on the decision variables of player $v$. We assume that $X \subseteq \mathbf{R}^{n}$ is a nonempty, closed (not necessarily compact) and convex set which represents the global constraints of all players $v=1, \ldots, N$, we therefore have

$$
X_{v}\left(x_{-v}\right):=\left\{x_{v} \mid g\left(x_{v}, x_{-v}\right) \leq 0, h_{v}\left(x_{v}\right) \leq 0\right\}
$$

Definition 1 (Generalized Nash equilibrium): $A$ vector $x^{*} \in X$ is called a generalized Nash equilibrium, or simply a solution of the GNEP, if $x^{*}$ satisfies

$$
\tau_{v}\left(x_{v}^{*}, x_{-v}^{*}\right) \leq \tau_{v}\left(x_{v}, x_{-v}^{*}\right) \quad \forall x_{v} \in X_{v}\left(x_{-v}^{*}\right)
$$

for each $v=1, \ldots, N$.

In order to solve the Single Objective Optimization (SOO) problem using Nash algorithms proposed in this paper, we introduce the concept of virtual game.

Definition 2 (Real and Virtual Games): In general, for any $i, j \in[1, \ldots, N]$ if $\tau_{i}=\tau_{j}=\tau$ or deal with a subset of $\tau^{\prime}$ such that $\sum_{i=1}^{N} \tau_{i}^{\prime}=\tau$ where $\tau_{i}^{\prime}>0$, we call above game as the Virtual Game; if $\exists i, j \in[1, \ldots, N]$ so that $\tau_{i} \neq \tau_{j}$ when $i \neq j$, we call above game as the Real Game.

With this definition, game theory can also be used to calculate the optimum solution of a SOO problem using the virtual game.

\subsection{Parallel Nash EAs/Das Hybrid Optimization Algorithms}

In this subsection, we introduce the NE based game theory to solve a MOO problem and provide EAs/DAs hybrid algorithms to capture a NE solution. 
Consider the two objective optimization problem defined as follows:

$$
\begin{aligned}
& \text { Player 1: } \min _{x_{1} \in X_{1}} \tau_{1}\left(x_{1}, x_{2}\right) \\
& \text { Player 2: } \min _{x_{2} \in X_{2}} \tau_{2}\left(x_{1}, x_{2}\right)
\end{aligned}
$$

In this game $N=2$, vectors $x_{1}$ and $x_{2}$ are sub-vectors of design variables $x=\left(x_{1}, x_{2}\right) \in X \subseteq \mathbf{R}^{n} . x_{1} \in X_{1} \subseteq \mathbf{R}^{n_{1}}$ and $x_{2} \in X_{2} \subseteq \mathbf{R}^{n_{2}}, n_{1}+n_{2}=n \geq N$. The NE is computed iteratively as follows:

As introduced in Nash theory, Player 1 optimizes the first criterion $\tau_{1}$ by modifying $x_{1}$, while $x_{2}$ is fixed by Player 2. Symmetrically, Player 2 optimizes the second criterion $\tau_{2}$ by modifying $x_{2}$ while $x_{1}$ is fixed by Player 1. Let's suppose that Player 1's optimization task is performed on population by using evolutionary algorithm whereas Player 2's optimization task is performed by using gradient-based method. The iteration procedure for building the NE using EAs/DAs hybrid algorithms can been summarized as

$$
x^{m}=H_{E A s / D A s}\left(x^{m-1}\right)
$$

where, iteration function $H_{E A s / D A s}$ are

$$
\left\{\begin{array}{c}
x_{1}^{(0)}=x_{1}^{m-1}, x_{2}^{(0)}=x_{2}^{m-1} \\
\text { Do } k=1, \ldots, \mathbf{K}^{\prime} \\
x_{1}^{(k)}=E_{\text {volutionary Algorithms }}^{\tau_{1}}\left(x_{1}, x_{2}^{(0)}\right) \\
\text { Enddo } \\
\text { Do } k=1, \ldots, \mathbf{K} \\
x_{2}^{(k)}=x_{2}^{(k-1)}-\lambda g_{\text {rad }}^{\text {player } 2}\left(x_{1}^{(0)}, x_{2}^{(k-1)}\right) \\
\text { Enddo } \\
x_{1}^{m}=x_{1}^{\left(\mathbf{K}^{\prime}\right)}, x_{2}^{m}=x_{2}^{(\mathbf{K})}
\end{array}\right.
$$

In this case,

$$
\begin{aligned}
& x_{1}^{m}=\left\{x_{1} \in \mathbf{R}^{n_{1}} \mid \tau_{1}=\inf _{x_{1} \in \mathbf{R}^{n_{1}}} \tau_{1}\left(x_{1}, x_{2}^{m-1}\right)\right\} \\
& x_{2}^{m}=\left\{x_{2} \in \mathbf{R}^{n_{2}} \mid \tau_{2}=\inf _{x_{2} \in \mathbf{R}^{n_{2}}} \tau_{2}\left(x_{1}^{m-1}, x_{2}\right)\right\}
\end{aligned}
$$

The NE is reached when neither Player 1 nor Player 2 can further improve their criteria. This hybridized EAs/DAs Nash algorithm is presented in Fig. 1. 


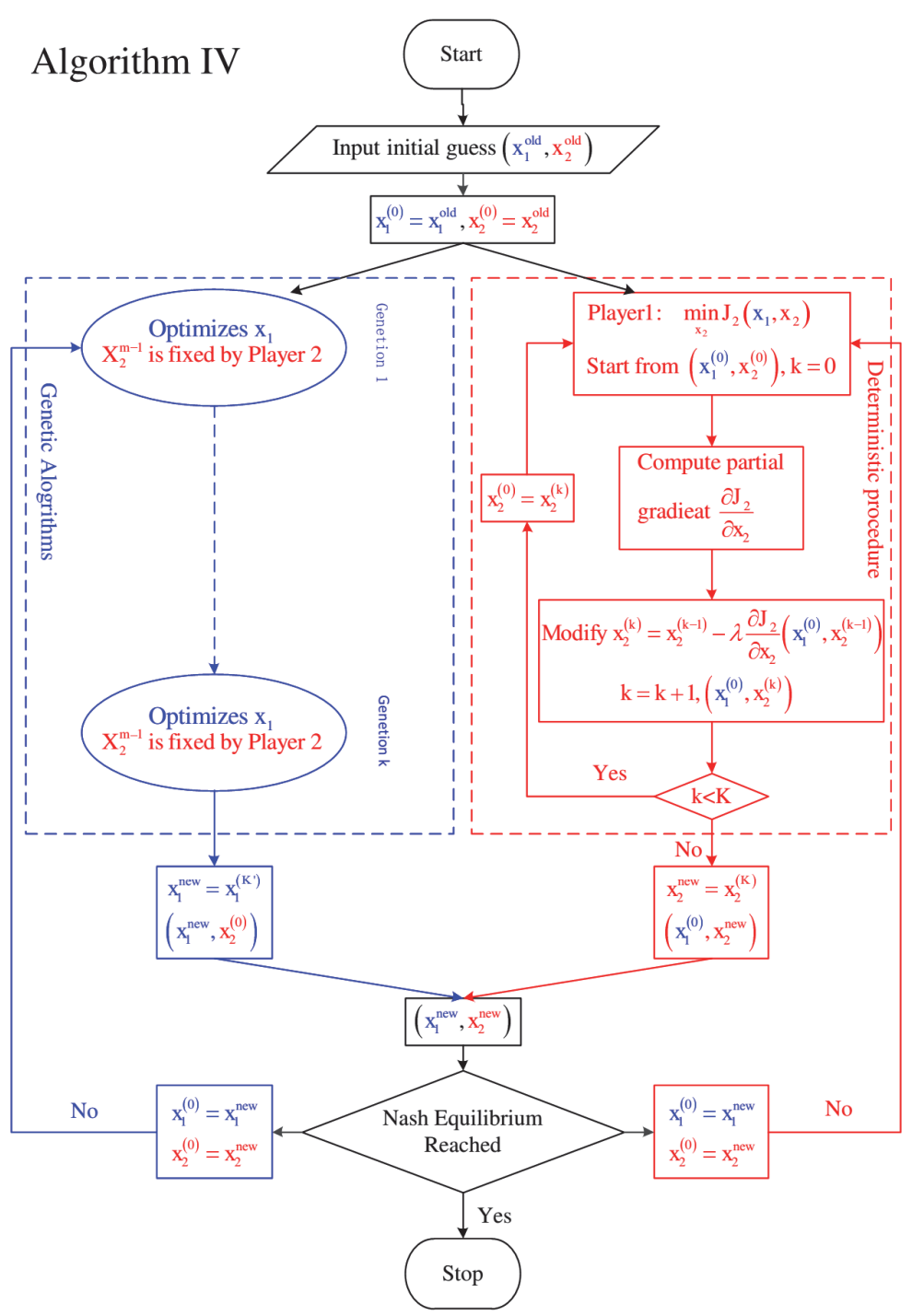

Fig. 1. Sketch of parallel Nash EAs/DAs hybrid algorithms.

The source terms in Eq. (1) are handled separately. Time integration is performed using two-stage Runge-Kutta algorithm and the resulting method is also second-order accurate in time.

\section{Application in Virtual Multi-Objective Aerodynamic optimization}

In this section, we consider drag minimization of a wing fuselage shape (see figure 2) at $M_{\infty}=0.785$, while lift coefficient $C_{L}$ is maintained. The flow is modeled by the 
compressible full potential flow with viscous boundary layer correction. This problem consists of minimizing a single objective function $\tau=C_{D}$ as follows

$$
\begin{aligned}
& \min _{\text {wing }} \tau=C_{D_{\text {wing }+ \text { fiselage }}} \\
& C_{L_{\text {wing }+ \text { fuselage }}}=\text { constant }
\end{aligned}
$$

Wing shape is defined by eight sections as shown in Figure 2. Each section is described by two Bezier curves, one for the suction side and the other for the pressure side. The section shape is determined by $2 \times 13$ control points, control points located at leading edge and trailing edge are fixed. In addition, dihedral position and twist angle of each section are also free to be modified in optimization. Thus, two sets of parameters are used, namely globally and locally. The global parameters of the wing shape are dihedral position and twist angle of each section. The local parameters of the wing shape are defined by wing sections, each section shape is determined by 22 control points, see Figure 2. Meanwhile, angle of attack is adjusted to perform lift constraint during optimization. Hence, there are 193 design variables in totally.
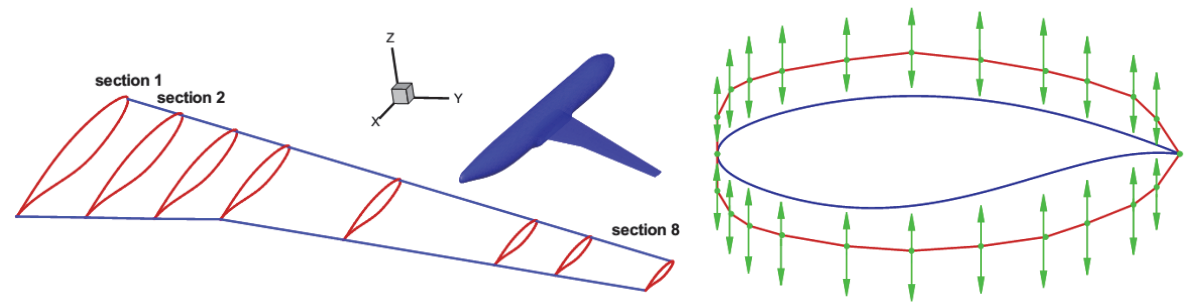

Fig. 2. Parameterization for civil transport wing fuselage configuration.

As mentioned in Ref. 10, the poor result was obtained by optimizing simultaneously local and global parameters. One may suppose that the mixing of global and local parameters leads to complex interactions among multi-modality. This suggests the use of virtual Nash games, as a way to couple the optimizations of local and global shape parameters. Indeed, Nash games are based on the concept of split of territories, which consist of splitting the design variables in two sets, each set being optimized independently by a so called player. Here, both players will have the same objective function, and then we refer to virtual Nash games.

$$
\begin{aligned}
& \text { Player1 }(E A s): \min _{\text {global parameters }} \tau=C_{D_{\text {wing }+ \text { fuselage }}} \\
& \text { Player } 2(E A s): \min _{\text {local parameters }} \tau=C_{D_{\text {wing }+ \text { fuselage }}}
\end{aligned}
$$

Such a strategy can reduce significantly the computational cost because each optimization is carried out in a subspace with lower dimension and they can be solved in parallel, since they are independent. Player 1 performs optimization by using EAs on the population of 16 global design variables, and player 2 performs optimization by using 
gradient-based optimization method (e.g. BFGS) since there are 176 design variables in optimization, in which the gradient is computed via finite difference. Thus, virtual Nash algorithm is used to solve optimization problem (10). Typically, any relaxation scheme quickly damps the high-frequency components of the error on a grid $^{11}$. Global design variables produce low-frequency changes on the shape, and locally ones produce highfrequency changes, so flow field analyzer of player 1 is implemented on coarse mesh, while player 2 is on fine mesh.

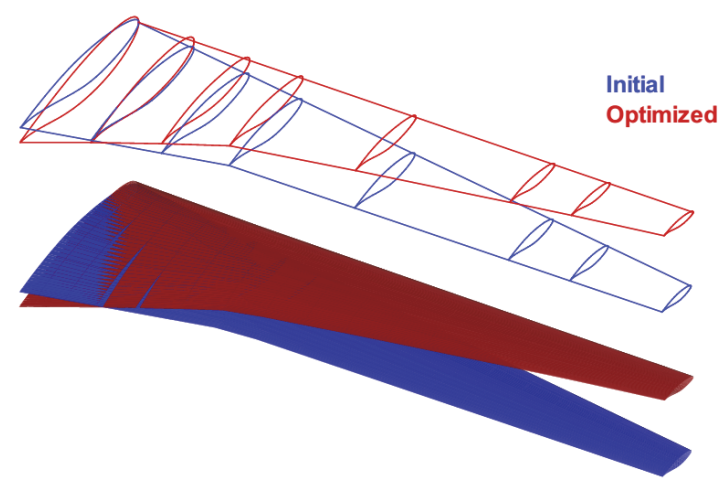

Fig. 3. Comparison of the shape for the baseline wing and the wing optimized by Nash game.

Table 1 Comparison of aerodynamic performances of baseline and Nash optimized wing fuselage configurations.

\begin{tabular}{|l|l|l|l|l|l|l|l|}
\hline & $\begin{array}{l}\text { Flight } \\
\text { condition }\end{array}$ & $M \frac{L}{D}$ & $C_{L_{W F}}$ & $C_{D_{W F}}$ & $C_{D_{I N D}}$ & $C_{D_{W A V E}}$ & $C_{D_{P D}}$ \\
\hline Baseline & $\begin{array}{l}M=0.785 \\
\alpha=2.625^{0}\end{array}$ & 20.55 & .5654 & .02160 & .01097 & .00359 & .00704 \\
\hline Optimized & $\begin{array}{l}M=0.785 \\
\alpha=1.920^{0}\end{array}$ & 25.35 & .5650 & .01749 & .01084 & .00021 & .00645 \\
\hline
\end{tabular}

The planform of wing is maintained during optimization, and the baseline wing is without twist and dihedral. The comparison of baseline and optimized wing shapes are shown in Figure 3. The use of virtual Nash games has been found more effective to couple local and global optimizations, yielding a better objective function value without increasing the computational cost. Aerodynamic performances of baseline and Nash optimized wing fuselage configurations are listed in Table 1 . The cruise factor $M * L / D$ is improved apparently from 20.549 to 25.35 due to drag coefficient $C_{D W F}$ of wing fuselage configuration is obviously reduced from 0.0216 to 0.01749 after optimization. Lift is maintained by adjusting angle of attack during optimization. Wave drag $C_{D W A V E}$ of wing fuselage configuration is evidently reduced from 0.00359 to 0.00021 , this can be reflected from pressure contours and pressure distributions on baseline and Nash optimized wings, i.e., Figure 4, where significant shock wave reduction is achieved on 
the suction side of Nash optimized wing. Profile drag $C_{D P D}$ is also reduced from 0.00704 to 0.00645 .

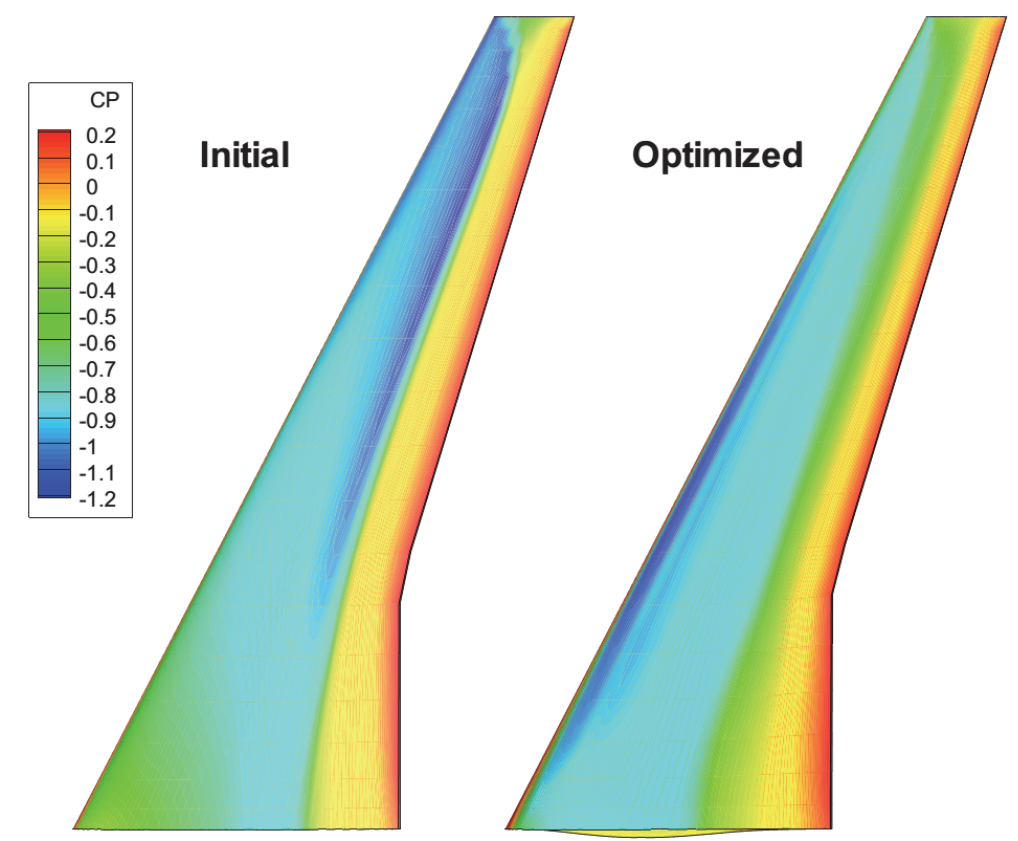

Fig. 4. Comparison of pressure contours on upper surface of baseline and Nash optimized wings.

\section{Conclusions}

An EAs/DAs hybrid algorithm for calculating a Nash Equilibrium is successfully used to solve aerodynamic design optimization problems, the successful application validates efficiency of algorithms in solving complex engineering optimization problem.

For the optimization problems with mixing of global and local design variables, smooth changes are made separately from high-frequency changes by using NE algorithms. The flow field due to smooth changes in the shape is solved on coarse grids, and the flow field due to high-frequency shape changes is solved on increasingly fine grids. This breaks the optimization procedure into a simultaneous of sub-optimization problems. The resulting optimization procedure is very efficient because the work on a particular scale is done on the appropriate grid.

\section{References}

1. J.F. Nash, Equilibrium points in n-person games, Proc. Nat. Acad. Sci. U.S.A. 36 (1950) 46-49.

2. J.F. Nash, Noncooperative games, Ann. Math. 54 (1951) 286-295.

3. Z. Tang, J. A. Désidéri, J. Périaux, Multi-criteria aerodynamic shape-design optimization and inverse problems using control theory and Nash games, J. Optimiz. Theory App., 1, 599-622 (2007). 
4. Z. Tang, W. Bai, J. Dong, Distributed optimization using virtual and real game strategies for multi-criterion aerodynamic design, Sci. China Ser. E, 51(11), 1939-1956 (2008).

5. Z. Tang, J. Dong, Couplings in multi-criterion aerodynamic optimization problems using adjoint methods and game strategies, Chinese J. Aeronaut., 22, 1-8 (2009).

6. D. S. Lee, L. F. Gonzalez, J. Periaux, K. Srinivas, Hybrid-game strategies coupled to evolutionary algorithms for robust multidisciplinary design optimization in aerospace engineering, IEEE T. Evolut. Comput., 15(2), 133-150 (2011).

7. A. Habbal, J. Petersson, M. Thellner, Multidisciplinary topology optimization solved as a Nash game, Int. J. Numer. Meth. Eng., 61(7), 949-963 (2004).

8. S. Li, Y. Zhang, Q. Zhu, Nash-optimization enhanced distributed model predictive control applied to the shell benchmark problem, Inform. Sciences, 170(2-4), 329-349 (2005).

9. G. Debreu, A social equilibrium existence theorem, Proc. Nat. Acad. Sci., 38, 886-893 (1952).

10. R. Duvigneau, Coupling local and global shape optimization in aerodynamic design, Research Report RR-7684, Centre de Recherche INRIA Sophia Antipolis, 2011.

11. S.B. Hazra, A. Jameson, One-shot pseudo-time method for aerodynamic shape optimization using the Navier Stokes equations, Int. J. Numer. Meth. Fl. 68 (2012) 564-581. 